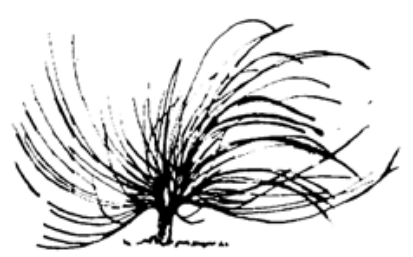

\title{
El desarrollo de habilidades académicas y emocionales adquiridas por el estudiantado con discapacidad durante el proceso de formación universitaria
}

\author{
Roxana Rodríguez Arayal \\ Universidad Nacional de Costa Rica \\ Heredia, Costa Rica \\ rodri156@gmail.com
}

\begin{abstract}
Resumen
Este ensayo ahonda en las habilidades emocionales y académicas que desarrolla el estudiantado con discapacidad durante el proceso de formación universitaria en la Universidad Nacional. La reflexión nace de la experiencia acuñada durante siete años de trabajar en un servicio de apoyo educativo ofrecido a esta población, así mismo se aborda las barreras de entorno a las que se enfrentan estos grupos de jóvenes. Por otra parte, se presentan los cambios que se requieren tanto en el ámbito de políticas institucionales como en la organización administrativa y curricular para hacer efectiva la inclusión social y educativa de esta población en la Universidad Nacional.
\end{abstract}

\section{(2) (1) (1) 89}

Recibido: 22 de marzo de 2016-Aprobado: 22 de setiembre de 2016

1 Maestría Académica de la Universidad de Minnesota, certificado en Desórdenes del Espectro Autista de la Universidad de Minnesota. Actualmente labora como académica en la Universidad Nacional, en la División de Educación Básica del Centro de Investigación y Docencia en Educación (CIDE) de la Universidad Nacional, Costa Rica 
Palabras clave: Educación inclusiva, discapacidad, universidad, servicios.

\begin{abstract}
This essay refers to the social and academic abilities developed by the students with disabilities during their university years. Reflecting on the subject is part of the experience gained while working on an educational in-service program for this group of students at the Universidad Nacional, Costa Rica; at the same time, this essay aims to talk about the barriers these students encounter while at the university and the political and structural changes the university needs to do to make effective the social and educational participation of these students.
\end{abstract}

Keywords: inclusive education, disability, university, services

\title{
Introducción
}

El proceso de inserción al contexto universitario implica que el estudiantado realice una serie de cambios y adaptaciones a sus estilos de vida; lo cual implica el desarrollo de destrezas y habilidades para alcanzar la autonomía en sus ambientes comunales y familiares; asimismo para adquirir el sentido de responsabilidad que le permita enfrentarse a la dinámica universitaria con una carga académica con mayor exigencia.

Este proceso no es diferente para el grupo de estudiantes que presentan una condición de discapacidad; por el contrario, los retos en el ámbito personal y social se unen a otros que tienen que enfrentar en el contexto universitario, tales como las condiciones de accesibilidad en el espacio físico y en la infraestructura, en los procedimientos administrativos, en el ámbito curricular, en el acceso a la información y la comunicación, entre otras situaciones cada vez más complejas.

La capacidad de enfrentar estas situaciones depende, en primera instancia, de su propio autoconcepto. En este sentido, la literatura identifica que el autoconcepto es un elemento importante, entendido "como la percepción que el individuo tiene sobre sí mismo, basada directamente en sus experiencias con los demás y en las atribuciones que él mismo 
hace de su conducta" (Shavelson, Hubner y Stanton, citados por Polo y López, 2011, p. 88). Así mismo, el autoconcepto puede ser visto de manera mutidimensional; es decir "se divide en académico y no académico estando constituido este último por componentes emocionales, sociales y físicos" (Elexpuru et al., 1992; García y Musitu, 2001, citado por Polo y López, 2011, p. 88).

En el trabajo desplegado en el proyecto UNA Educación de Calidad, se ha comprobado que el desarrollo de habilidades, tanto académicas como socioemocionales de la población con discapacidad, es esencial para la permanencia y finalización de los estudios en la Universidad Nacional. En esta línea, se entiende por habilidades socioemocionales como:

Un conjunto de capacidades, conductas y estrategias, que permiten a la persona construir y valorar su propia identidad, actuar competentemente, relacionarse satisfactoriamente con otras personas y afrontar, de forma positiva las demandas, retos y dificultades de la vida, lo que posibilita su ajuste y adaptación, su bienestar personal e interpersonal, y vivir una vida más plena y satisfactoria. (Monjas, 2002, p. 37)

Es importante indicar que el desarrollo de las habilidades emocionales durante la formación universitaria es un elemento determinante para alcanzar el éxito tanto en el ámbito académico como en el social, la cual va a permitir una futura inserción laboral. Este, en el caso de la persona con discapacidad, es un tema importante, pues tal como lo menciona el Plan Nacional de Inserción Laboral para la Población con Discapacidad en Costa Rica (MTSS, 2012, según el Décimo Sétimo Informe del Estado de la Nación, 2011, con base en la ENAHO 2010), "el $63,7 \%$ de las personas con discapacidad se encuentran inactivas laboralmente" (p.19).

La formación universitaria y las habilidades sociales que el estudiantado desarrolle van a potenciar futuros procesos laborales que, sin duda alguna, serán esenciales para esta población. Sin embargo, dentro de la diversidad de este grupo de estudiantes, existe un conglomerado significativo compuesto principalmente por estudiantes con discapacidad de primer ingreso, que se enfrentan por primera vez a situaciones en las que deben tomar decisiones y autogestionar las formas de apoyo 
y seguimiento que requieren. Estas experiencias, si bien algunas veces complejas y estresantes, les permiten construir su identidad personal de adulto joven; mejorar su actitud hacia los retos y adaptarse positivamente a la dinámica universitaria $\mathrm{y}$, consecuentemente, en otras áreas de la vida.

Ahora bien, no solo el desarrollo de las habilidades emocionales y sociales es necesario, el estudiantado con discapacidad se enfrenta a un sistema educativo que exige de ellos y ellas resultados académicos, por lo cual es necesario desplegar nuevas estrategias cognitivas y metacogntivas para el aprendizaje específico de su área profesional, que les permita avanzar en sus carreras. Por otra parte, se requieren las destrezas básicas de lectura y escritura del idioma español (redacción, síntesis, análisis entre otras) y del uso de recursos tecnológicos para un mejor desempeño.

Este ensayo pretende reflexionar sobre las habilidades socioemocionales tanto como las académicas, desarrolladas por estudiantes en condición de discapacidad en el contexto de la Universidad Nacional de Costa Rica, durante su formación profesional. También, se procura presentar las barreras del entorno a las que se enfrentan estos grupos de estudiantes; adenás, pretende dar orientaciones en el ámbito de políticas institucionales y en la organización administrativa y curricular, para lo cual considera los principios humanistas que han identificado a esta casa de enseñanza, requerimiento al realizar acciones concretas para garantizar no solo el acceso, sino la permanencia y graduación de este grupo de la diversidad estudiantil.

\section{Reflexión en torno al acceso del estudiante con discapacidad a la Universidad Nacional}

El acceso de estudiantes con condición de discapacidad o con necesidades educativas ha venido en aumento en la Universidad Nacional; al respecto, en el año 2012 se matricularon 86 estudiantes. En el 2013, 70 estudiantes, en el 2014 la cifra aumentó a 110 y en el 2015 fueron 117. Estas cifras incluyen estudiantes con discapacidad y necesidades educativas.

El aumento responde a un fenómeno que se ha dado en múltiples universidades, en las cuales se han reportado incrementos importantes en las matrículas de esta población (Alcedo, Aguado, Real, González 
y Rueda, 2007; Guillamón y Rodríguez, 2010). Posiblemente, esto responde a un proceso social de reconocimiento de la discapacidad y de los derechos educativos de estas personas, aunque también existen cuestionamientos sobre la tasa de permanencia y culminación de los estudios superiores. Si bien, estos temas son importantes de analizar, el objetivo de este ensayo no va en esta línea, sino en reflexionar acerca de los aprendizajes de estudiantes con discapacidad que sí permanecen en la universidad.

En relación con el ingreso de la población con discapacidad a la universidad, se han desarrollado una serie de servicios de apoyo para el estudiantado. Estos servicios juegan un papel muy importante al ofrecerles un espacio de orientación y acompañamiento. En el caso de Europa y Estados Unidos, existen servicios generales (dirigidos a la población total) y específicos (los cuales responden a las características específicas del individuo) (Abad, Alvaréz y Castro de Paz (s. f.). En relación con los servicios específicos, los autores referidos anteriormente los describen como:

Un servicio o programa destinado a la atención de estudiantes con discapacidad, que se encarga de proveer una estructura de apoyo, coordinar los servicios prestados, apoyos, accesibilidad, recursos...que estos alumnos necesitan. (p. 8)

La Universidad Nacional de Costa Rica, al igual que otros centros de enseñanza superior del país, desarrolla desde 1998 el proyecto UNA Educación de Calidad que ofrece un servicio de apoyo educativo directo a esta población estudiantil con discapacidad, el cual incluye tanto servicios generales como específicos; tales como apoyo a académico, apoyo en el acceso a la información y al espacio físico, trabajo de pares, búsqueda de materiales, entre otros. Nuestra experiencia nos indica que parte del apoyo consiste en proveer un espacio para la socialización, el compartir y el sentir del estudiantado. Por eso es común tener espacios informales para conversar y, de esta forma, orientarles, comprendiendo no solo su situación estudiantil, sino su realidad social y personal.

Aunado al ingreso de estos grupos de estudiantes en los contextos universitarios y a los servicios que se ofrecen en las universidades, específicamente los proveídos por el proyecto mencionado, surge la 
necesidad de identificar las habilidades desarrolladas por la población durante su proceso de formación y las barreras del entorno que limitan la participación dentro de la universidad. En esta línea surgen interrogantes como las siguientes:

1. ¿Cuáles habilidades personales (académicas y emocionales) desarrolla el estudiantado con discapacidad durante sus años en la universidad?

2. ¿Cómo apoyar el desarrollo de habilidades y destrezas (académicas y emocionales) en este estudiantado para asegurar la permanencia y éxito de sus procesos de aprendizaje?

3. ¿A cuáles barreras del entorno se enfrentan durante su desarrollo profesional?

Al indagar sobre estos cuestionamientos con estudiantes con discapacidad se han identificado luchas personales, así como con las barreras que el entorno continúa manteniendo, entre estas: visiones de sus familiares sobre la condición de discapacidad y sus tendencias a la protección o al irrespeto de sus decisiones, falta de apoyo de sus docentes y un entorno social y académico que no siempre se adapta a sus condiciones personales, tales como inaccesibilidad en los sistemas universitarios asociados al aula virtual, sistemas de becas, entre otros. Con el afán de superar estos retos, sobresalen entre otras cualidades personales la flexibilidad, la autogestión y el esfuerzo.

Para ejemplificar estas experiencias, se les solicitó a un grupo de quince estudiantes que narraran en un grupo focal y en conversaciones posteriores sus vivencias pasadas y actuales con respecto a la experiencia en la universidad. Así mismo, completaron una entrevista que ahondaba en este tema. Algunas palabras que surgen de la reflexión de cuando iniciaron su proceso universitario son: estrés, dificil, adaptación, ilusión, emoción, locura, temor, incertidumbre, cansancio, tristeza, desesperación, lucha. Estas palabras en su mayoría reflejan emociones o experiencias negativas, más que positivas, que responden a las necesidades de adaptarse a un entorno nuevo y autogestionar sus vidas tanto a nivel personal como académico. Sin embargo, al preguntarles sobre sus experiencias actuales, las palabras se han transformado en emociones o experiencias positivas, entre estas resaltan: logros, transiciones, grandes sueños, satisfacción, apertura a nuevos aprendizajes, 
motivación, estrés y frustración, ser más fuerte, sentirse profesional, segura de si misma y compromiso. Como puede verse, los estudiantes van cambiando la mayoría de sus percepciones sobre la experiencia inicial, estos cambios son atribuidos a sus propias luchas, a la experiencia del proceso de adaptación y aprendizaje del contexto de la universidad; y finalmente al gran deseo de desarrollar su potencial profesional.

En muchos casos, la universidad es la primera experiencia de autonomía, e independencia para estos grupos de estudiantes. Como personas adultas deben enfrentar retos que anteriormente eran sus padres o madres quienes los asumían; el alejarse de su familia (geográficamente o simbólicamente), les permite reencontrarse consigo y desarrollar su identidad personal. La siguiente narración es un ejemplo de esto:

Es duro adaptarse cuando uno viene de una zona rural, en especial dependiendo de la familia. Tres años después, sentirse bien, estar aquí quita la vergüenza, y los miedos. No hay palabras para expresar lo que uno siente. Tomar la decisión y que nadie se meta. No hay palabras... En lo académico, mi carrera no es lo que quiero, pero poder tomar la decisión de cambiarme sin la presión de mi familia, es increíble, por fin puedo luchar solo. (Comunicación personal, octubre 25, 2015)

Al escuchar esta y otras experiencias del estudiantado se visualiza lo valioso que es el reconocimiento de esta población dentro de un marco social o de derechos humanos, donde se conciben como personas autónomas, individuales y poseedoras de derechos. Hace 30 años, pensar en estudiantes con discapacidad que participaran de la vida universitaria era en muchas medidas imposible; sin embargo, estos cambios surgen de la lucha de la población con discapacidad para que exista un verdadero reconocimiento de sus derechos humanos. Estos esfuerzos fueron iniciados por organizaciones de personas con discapacidad en la década de los años 70 y desde entonces los cambios paradigmáticos han evolucionado desde concepciones basadas en el déficit hasta la visión social actual enfocada en el reconocimiento de la diversidad y las barreras del entorno que definen, en gran medida, la condición de discapacidad (Fontana, Vargas y Quirós, 2015).

El cambio de visión de la discapacidad influyó grandemente en la atención educativa que se le ofrece a esta población, la cual 
gradualmente ha venido evolucionado desde una visión rehabilitadora, centrada en el déficit y que buscaba el abordaje educativo desde la experticia de especialistas, hacia una visión de educación inclusiva que centra sus esfuerzos en reconocer al ser humano; sus derechos, intereses, potencialidades y necesidades. Así mismo, busca identificar en el ambiente educativo y social las barreras para minimizarlas; y de esta forma promover la participación de la población en equidad de condiciones.

En esta línea han surgido propuestas sobre el modelo de centro educativo y los principios que deben regir el accionar educativo. Estos principios fueron ratificados en la Declaración de Salamanca, los cuales son:

a. Que todos los miembros del centro se sientan partícipes del mismo, aceptados, seguros y bienvenidos.

b. Aumentar la participación de todos para que logren el máximo aprendizaje y desarrollo personal y social, entre otras cosas, identificar las barreras presentes en la organización del centro y del currículo, así como asumir el compromiso de su eliminación progresiva.

c. Dedicar una atención especial a grupos e individuos con mayor riesgo de exclusión.

d. Crear culturas inclusivas en el sentido de construir una comunidad segura, acogedora y estimulante y colaboradora, en la que cada docente es considerado como el fundamento primordial para que todo alumno alcance mayores niveles de logro.

e. Elaborar políticas inclusivas que tienen que ver con la línea estratégica que cada centro decide, planifica y organiza para regular la vida del centro con el objetivo que mejore el aprendizaje y la participación de todos los componentes. (Jiménez, 2007, p. 30)

Estos principios orientadores tienen, en esencia, la intencionalidad de que cada organización gestione cambios en un marco de respeto a la diversidad humana y que los aprendizajes se desarrollen desde este reconocimiento. El impacto social es grande, pues retoma ideales como la participación, la construcción de una sociedad segura, inclusiva y colaboradora. Para todos los centros educativos existen retos diferentes en la forma en que estos principios pueden ser operacionalizados en su propia cultura organizativa, y los centros de educación superior no 
se escapan de esta realidad. Sin embargo, la experiencia acuñada ha demostrado que la comunicación, participación y democratización de las conversaciones sobre discapacidad y accesibilidad son necesarias para lograr una visión conjunta y un servicio educativo que responda a lo que estos grupos de estudiantes desean y necesitan.

\section{Habilidades desarrolladas por estudiantes con discapacidad en la Universidad Nacional}

Aprender a ser y aprender a convivir se convierten en competencias cada día más relevantes. La vida misma y las experiencias que en ella tenemos, son las que nos permiten, como seres humanos, convivir de una forma pacífica y plena en sociedad. La comprensión del sentir del individuo tiene implicaciones significativas en los contextos de la vida en los que se relaciona, tales como procesos de formación y el quehacer profesional. Por esto cada día se vuelve más importante la reflexión sobre la inteligencia emocional, entendida como:

La capacidad de motivarnos a nosotros mismos, de pensar en el empeño a pesar de las posibles frustraciones, de controlar los impulsos, de deferir las gratificaciones, de regular nuestros propios estados de ánimo, de evitar que la angustia interfiera en nuestras facultades racionales. (Goleman, 1995, p. 26)

Dentro del concepto de la inteligencia emocional, se conciben las habilidades emocionales, sean estas intrapersonales (autoconocimiento, autoestima) como las interpersonales (habilidades sociales, empatía) como elementos que componen el concepto.

Las habilidades intra e inter personales se desarrollan a lo largo de la vida, por medio de procesos vividos en los contextos familiares, educativos y sociales. Sin embargo, para las personas con discapacidad, el perfeccionamiento de estas habilidades emocionales para enfrentar retos, construir su identidad y enfrentar dificultades de la vida toma connotaciones diferentes, pues están mucho más expuestos a la sobreprotección y a sus consecuencias; por lo tanto, puede que estas se desplieguen en un momento posterior al resto de la población. La sobreprotección de la población con discapacidad no es un tema nuevo, la 
bibliografía apunta a este fenómeno que se plasma en muchas ocasiones en los contextos familiares. En esta línea, García y Fernández (2005) hacen una reflexión sobre la inclusión de las personas con discapacidad que se polariza entre la igualdad y la diferencia, y mencionan sobre la sobreprotección que:

se constituye en una respuesta a las condiciones sociales que históricamente han constituido el ambiente en que las familias y las personas que presentan discapacidad han debido desenvolverse. $\mathrm{Su}$ conformación se constituye como consecuencia de una construcción históricamente modelada, esto se explica porque la sociedad se ha relacionado con el mundo de la discapacidad negando su existencia. ...(Al interior del mundo de la discapacidad la negación de la persona aparece como una distinción compartida. Esta negación toma diferentes formas entre ellas todas las formas de discriminación, invalidación y exclusión social). (p. 242)

La cita anterior apunta a una experiencia compartida de la familia entorno a la discriminación, invalidación y exclusión social, por lo tanto, la sobreprotección en muchas ocasiones surge como una respuesta de protección que, aunque bien intencionada, puede limitar en gran medida el desarrollo de habilidades sociales y personales de las personas con discapacidad. Al ser el contexto universitario un espacio que promueve la independencia de la totalidad de estudiantes de sus contextos familiares, es común ver que paulatinamente y a través de los años estudiantes con discapacidad consiguen desarrollar un empoderamiento personal; lo que permite que se visualicen como personas responsables de las decisiones de sus vidas, con mayores habilidades emocionales y académicas que les facilita continuar sus procesos educativos. Tanto las habilidades emocionales como las académicas son autoconstruidas, probadas y adaptadas por el estudiantado con discapacidad en la universidad. Al conversar con jóvenes sobre el desarrollo de estas durante su proceso universitario, resaltan las siguientes: 


\section{Habilidades emocionales}

- Paciencia, tolerancia (porque tengo que lidiar con las personas)

- Inteligencia emocional

- Escucha a las demás personas

- Positividad

- Asertividad con los otros seres

- Lucha por mis ideales

- Mejora de la autoestima

- Mejora de mis relaciones interpersonales

- Enfrentamiento a cosas nuevas

\section{Habilidades académicas}

- Mayor capacidad para analizar las cosas a nivel académico, pienso en otras formas de resolver las cosas

- $\quad$ Autogestión, proactividad; por ejemplo, me comuniqué con mis docentes desde antes del inicio del semestre. Yo les envié correos y les dije qué necesitaba. No esperé el mismo día de la clase, sino que desde antes sabían y podían asumir el reto. Así no dependo de nadie

- Mejora en la redacción de documentos

- Orden

- Más responsabilidad en mis labores

Continuando con la conversación sobre cuáles experiencias les han permitido desarrollar estas habilidades, mencionan; entre otras

- "enfrentarse a situaciones nuevas y desconocidas"

- " "el deseo de aprender y superarse"

- "vivir de manera independiente y tener mayor autonomía"

- "al independizarme de mi familia"

- "las vivencias en la universidad y las consecuencias de esas experiencias"

- "los diversos altibajos me ha enseñado y ayudado a mejorar para salir adelante" (comunicación personal, 27 de octubre, 2015) 
Al tratar de interpretar las habilidades desarrolladas, se evidencia que la vida universitaria las ha potenciado. Al conversar con esta población es claro que existe una conciencia sobre la importancia de estas habilidades para lograr una permanencia dentro de la universidad.

En esta línea, los estudios apuntan que los estados emocionales tienen una estrecha relación con los procesos cognitivos (García, 2012; Goleman, 1995), por lo cual la oportunidad de participación en la universidad, en sí misma, produce un efecto positivo y sostenido en la vida de las personas en condición de discapacidad y, consecuentemente, en muchos casos les permite potenciar aquellas habilidades académicas que requieren ser mejoradas para lograr su permanencia.

Pues bien, si la participación en el ambiente universitario produce un efecto positivo en la población con discapacidad, se requiere, entonces, identificar las barreras del entorno y tomar acciones, especialmente desde las líneas estratégicas de la universidad. Cada vez se hace más necesario crear una política inclusiva que realmente visualice las necesidades y potencialidades de la población estudiantil con discapacidad. No basta con ajustes en el examen de admisión y aumentar la matrícula de estudiantes con discapacidad, deben existir acciones concretas que estimulen la permanencia y conclusión de sus estudios. Esta postura es congruente con lo que indica el Estatuto Orgánico en el apartado de principios que rigen el quehacer universitario, más específicamente el principio de inclusión que plantea "La inclusión de los sectores menos favorecidos por razones económicas, culturales o por discapacidad, se garantiza mediante una oferta académica, políticas de admisión y programas de becas especialmente dirigidos a esos grupos“ (Universidad Nacional, Estatuto Orgánico, Principios, 2015).

\section{Las barreras del entorno y la respuesta universitaria}

Desde la promulgación de la Convención de los Derechos Humanos para las personas con discapacidad en el 2006, se visualizan las barreras del entorno como un elemento importante en la definición de la discapacidad, y se definen como aquellas condiciones actitudinales o de acceso que obstaculizan la participación de las personas con discapacidad en los contextos sociales. La identificación de las barreras en el entorno constituye un elemento muy importante de análisis, pues permite realizar cambios conscientes en nuestras decisiones políticas, 
metodológicas y actitudinales orientadas a realizar acciones para la auténtica participación de la población con discapacidad en la comunidad.

Al reflexionar sobre los tipos de barreras identificados por estudiantes que comentaron sus experiencias, las barreras actitudinales son mucho más frecuentes e impactan en mayor escala las vivencias; incluyen los ambientes tanto familiares como universitarios. Entre ellas están:

\section{Actitudinales}

- $\quad$ "La comunicación con la profesora es difícil, pues es muy estricta y cree que siempre tiene razón".

- $\quad$ "Mala actitud de algunos compañeros que se expresan de forma negativa sobre uno. En los trabajos en grupo, a veces es incómodo pues no hay sensibilización de la gente".

- $\quad$ "En mi casa me dicen 'usted porque toma decisiones solo, si usted no ve' entonces, como uno va a hacer las cosas solo con ese apoyo".

- "A veces en su afán de protegerme, se meten en la toma de decisiones (familia)".

- "Mi mamá me dice que guarde el bastón, que yo veo" (comunicación personal, 30 de octubre, 2015)

\section{Acceso al entorno}

"La información no es accesible, especialmente en las referencias. Es difícil citar APA".

"El aula virtual no es accesible".

"El sistema de matrícula no nos favorece y realmente somos personas interesadas". (comunicación personal, 30 de octubre, 2015)

\section{Metodológicas}

- "Hay retos y uno se la va jugando, ningún profesor se sienta a decirme esto o lo otro para mejorar".

- "No hay una claridad de cómo evaluarnos".

- "El método de estudio es todo auditivo y a veces eso cansa". (comunicación personal, 30 de octubre, 2015) 
A raíz de las barreras encontradas y de otras que existen, surge la interrogante sobre los apoyos que este grupo de la diversidad requiere para mejorar su inserción y permanencia en la universidad. Considerando la experiencia acumulada con los años de trabajar con esta población en la UNA, se considera que se requiere desplegar acciones para alcanzar una educación inclusiva.

Política universitaria: se requiere la construcción de una política universitaria en la cual la discapacidad, al igual que el enfoque de género, se convierta en un eje transversal que incida en todo el quehacer académico. Debe existir una agenda institucional que promueva y visibilice la diversidad de la persona con discapacidad como un ente activo de la comunidad universitaria. Vinculada a la política, es necesaria una agenda que, entre otros puntos, inicie un proceso de concienciación de la comunidad universitaria sobre la inclusión y permanencia de la población con discapacidad desde el enfoque de derechos.

Quehacer universitario: desde la docencia, la investigación y la extensión se requiere que el eje de discapacidad sea parte de la formación de personas en múltiples disciplinas. Estas nuevas profesiones necesitan ser conocedoras de los derechos de esta población. Por ejemplo, se requieren personal de bibliotecología que sepa atender a los sujetos usuarios de los centros de documentación en comunidades, secretarías que puedan atender a personas con discapacidad en sus funciones laborales, personal de las comunicaciones sociales que tenga voluntad de cambiar las visiones de lástima o de superhéroes; que muchas veces es la tónica en la que las noticias presentan la discapacidad. Para construir una sociedad que reciba y visualice a las personas con discapacidad como ciudadanos y ciudadanas, se requiere calar desde el quehacer y acción social universitaria.

Gestión organizacional: se requiere la construcción de un programa institucional que desde el enfoque de Derechos Humanos apoye la permanencia de estudiantes en condición de discapacidad y que maximice los recursos existentes, tanto desde el componente pedagógico como desde el administrativo. Este programa debe articular de una forma adecuada los múltiples esfuerzos que actualmente se realizan para atender a la población. Por otro lado, se demanda una orientación vocacional que oriente a estos grupos de jóvenes hacia carreras que potencien sus habilidades y deseos profesionales. 
Vida estudiantil: se requiere la promoción de la participación en espacios relacionados con la vida estudiantil; tales como en las asociaciones de estudiantes, ferias estudiantiles, voluntariados, entre otros.

Investigación: se requiere analizar, entre otros datos, los obtenidos durante el proceso de admisión de a la universidad e identificar el porcentaje de permanencia de esta población con el fin de tomar medidas que promuevan la culminación de sus estudios. Así mismo, es necesario apoyar la docencia universitaria guiada desde los resultados que la investigación pueda arrojar. Finalmente, como ente social, la investigación universitaria en discapacidad, retomando los referentes teóricos más actualizados sobre el tema, debe promulgar acciones orientadas al respeto y reconocimiento al derecho a la participación social de esta población.

Para que existan todos estos cambios que permitan enfrentar las barreras del entorno, es necesaria una actitud inclusiva, positiva y responsable. Ninguno de los planteamientos hechos hasta ahora se consiguen de la noche a la mañana, pero así como hace algunos años la población que tenía acceso a la educación superior era pequeña y ha ido lentamente aumentando, así los esfuerzos que se inicien en el presente pueden dar frutos significativos a futuro. Estos cambios se necesitan para que la sociedad perciba y potencie las habilidades de las personas con discapacidad en múltiples contextos sociales.

Los aspectos mencionados con respecto a los cambios por realizar a nivel universitario para que exista, verdaderamente, una educación inclusiva, son congruentes con los retomados por Vargas, Fontana y Quirós (2015, citando a Lissi et al., 2009) al mencionar que se requieren las siguientes condiciones para que la universidad pueda ser considerada inclusiva:

a. políticas al interior de la universidad que promuevan la inclusión, así como con normativas y reglamentos;

b. acceso a los programas de estudio y desarrollo de actividades de sensibilización sobre la inclusión y la equidad de estudiantes con discapacidad;

c. orientación y asesoría a las y los docentes sobre las necesidades $\mathrm{y}$ adecuaciones requeridas;

d. incorporación de la temática de discapacidad en la malla curricular de las distintas carreras que se ofertan; 
e. aspectos en relación con la infraestructura, la accesibilidad física a todos los servicios y apoyos tecnológicos;

f. adaptaciones para la participación estudiantil en la vida universitaria;

g. programa de pares estudiantiles de apoyo;

h. aspectos relacionados con la investigación para optimizar las intervenciones. Dichos aspectos deben darse con "una mirada de la discapacidad en que las personas son valoradas y reconocidas como cualquier otro miembro de la comunidad universitaria". (p. 12)

Si bien es cierto, las posiciones presentadas son recurrentes a las que la teoría aporta, la necesidades siguen existiendo, las respuestas a estas inquietudes no se han resuelto completamente. Sin embargo, la población matriculada sigue incrementando; por lo tanto, continúa siendo relevante y necesaria esta reflexión.

Al trabajar con la población con discapacidad, se ha mostrado que la inclusión y participación es la mejor forma de que las personas con discapacidad puedan crecer como individuos. Hemos visto estudiantes evolucionar y ser personas más responsables consigo mismas y con las demás, más conocedoras de sus derechos y responsabilidades, más empoderadas y felices. Si la experiencia al trabajar con esta población ha enseñado algo, es que vale la pena apostar por una educación inclusiva, pues los resultados en el desarrollo de habilidades sociales y académicas son superiores a cualquier expectativa. El estudiantado con discapacidad no es especial como muchas veces las construcciones sociales lo han establecido, son personas con virtudes y aspectos por mejorar que requieren que abramos los espacios y que se les permita reencontrarse con sus vidas. Este es el principal reto y la gran oportunidad.

\section{Referencias}

Abad, M., Alvaréz, P., Castro de Paz, J. (s. f.). Apoyo a la integración de estudiantes con discapacidad en la enseñanza universitaria: Algunas medidas y propuestas de actuación orientadora. Universidad de la Laguna. España.

Alcedo, M., Aguado, A., Real, S., González, M. y Rueda, B. (2007). Una revisión actualizada de la situación de estudiantes con 
discapacidad en la universidad. Anuario de Psicología Clínica y de la Salud, 3, 7-18.

Elexpuru, I., Garma, A., Marroquín, M. y Villa, A. (1992). Autoconcepto y educación. Bilbao: Servicio Central de Publicaciones.

García, A. y Fernández, A. (2005). La inclusión para las personas con discapacidad: La igualdad y la diferencia. Revista Ciencias de la Salud, 3(2), 235-246.

García, J. (2012). La educación emocional: Su importancia en el proceso de aprendizaje. Revista Educación, 36(1), 97-109.

Goleman, D. (1995). Inteligencia emocional. Buenos Aires: Javier Vergara.

Guillamón, J. y Rodríguez, V. (2010). Atención y orientación a los estudiantes con discapacidad en la UNED. Revista española de Orientación y Psicopedagogía, 21(2) 391-400.

Jiménez, M. (2007) Propuestas curriculares para responder a la diversidad del alumnado de primaria desde contextos inclusivos. Contextos educativos, 10, 29-44.

Fontana, A., Vargas, M. y Quirós, M. (2015). Construcciones sobre discapacidad y necesidades educativas e implicaciones en la respuesta educativa del estudiantado de la Universidad Nacional de Costa Rica (UNA). IX Jornadas Cientificas de Investigación sobre Personas con Discapacidad, España. Recuperado de http:// sid.usal.es/ficheros/revista-siglo-cero-2015-anejo-1.pdf

Ministerio de Trabajo y Seguridad Social. (2012). Plan Nacional de inserción laboral para la población con discapacidad en Costa Rica. San José: PNUD.

Polo, M. y López, M. (2011) Autoconcepto de estudiantes universitarios con discapacidad visual, auditiva y motora. Revista Latinoamericana de Psicología, 44(2), 87-98.

Universidad Nacional. (2015). Estatuto Orgánico. Gaceta extraordinaria N 3-2016.

Vargas, M. Fontana, A. y Quirós, M. (2015). Necesidades de actualización de la población académica para la atención a estudiantes con discapacidad en la Universidad Nacional, Costa Rica. IX Jornadas Científicas de Investigación sobre Personas con Discapacidad, España. Recuperado de http://sid.usal.es/ficheros/revista-siglo-cero-2015-anejo-1.pdf 